\title{
Cephalic Duodeno-Pancreatectomy with Pancreatic-Gastric Anastomosis with Double Purse String, in Patient with Lithiasis and Tumoral Jaundice - Case Report
}

\author{
Tudor A, Molnar C, Nicolescu C, Rosca C, Tudor Bianca, Butiurca VO, Copotoiu C \\ University of Medicine and Pharmacy Tirgu-Mures
}

Introduction: One of the most feared complications after cephalic duodeno-pancreatectomy remains pancreatic fistula. In recent years, various methods of pancreatico-digestive reconstruction were performed in order to reduce the rate of pancreatic fistula. One of these methods is pancreatico-gastric reconstruction by using two purse string threads.

Case report: We present in this article a patient with jaundice with mixed etiology: tumoral and lithiasic. Subjectively, the patient accused sclerose-skin-jaundice, right upper quadrant and epigastric pain, nausea and vomiting. Computed tomography revealed dilatation of intraand extrahepatic bile ducts, a dilated Wirsung duct and a tumor at the biliopancreatic confluence, leading to a suspicion of vaterian ampulom. Upper endoscopy revealed a tumor protruding in the descending duodenal segment. Intraoperatively a tumor suggestive of vaterian ampulom and duct stones was shown. Surgical treatment consisted of coledocolitotomy, cephalic duodeno-pancreatectomy with pancreatic-gastric anastomosis, performed by using two purse string threads. The postoperative evolution was favorable.

Conclusion: Pancreatico-gastric anastomosis using two purse string threads is a simple, safe and quick procedure, avoiding the application of sutures through the pancreatic parenchyma and thus reducing the rate of pancreatic fistula.

Keywords: cephalic duodeno-pancreatectomy, pancreatic fistula, double purse string, pancreatico-gastric anastomosis

Received: 07 October 2014 / Accepted 29 October 2014

\section{Introduction}

One of the worst complications after cephalic duodenopancreatectomy remains pancreatic fistula [1,2]. Several publications have shown that the incidence of this complication is larger in the case of the so-called "soft" pancreas $[3,4]$. Considered the "Achilles heel" of this largescale intervention, pancreatico-digestive anastomosis has known many improvements in recent years, both in terms of anastomosis of the pancreas with stomach or jejunum. Numerous studies have compared the postoperative complications after the two types of anastomosis. Some have not found differences in statistical terms on incidence of pancreatic fistula $[5,6,7,8]$, while others have shown a lower incidence of complications after pancreatic anastomosis with the stomach $[7,9]$. In the recent years several techniques have described the anastomosis of the pancreas to the stomach, each trying to reduce as much as possible the incidence of pancreatic fistula. In this paper we report a patient with jaundice with lithiasic and tumor etiology, who underwent cephalic duodeno-pancreatectomy, with a pancreatico-gastric reconstruction with double purse string to the posterior gastric wall.

Correspondence to: Calin Molnar E-mail: molnar.calin@yahoo.com

\section{Case report}

We present the case of a patient aged 68 years, admitted in the Surgical Clinic No. I, Emergency County Hospital Tirgu Mures in emergency conditions, with the diagnosis of jaundice and suspected cephalic pancreatic tumor, being refrerred from the Clinic of Infectious Diseases, where the viral etiology of jaundice was ruled out. Patient complaints were jaundice, nausea, vomiting, abdominal pain located in the epigastric region and right hypochondrium. From the patient history, we retain a laparoscopic cholecystectomy for gangrenous cholecystitis made three years ago. Abdominal ultrasound was performed in emergency conditions, indicating dilated extra-and intrahepatic biliary tract and suspicion of a cephalic pancreatic tumor. Abdominal computed tomography showed a tumoral process in the biliary-pancreatic confluence suggestive of a tumor located at the ampulla of Vater, moderate dilatation of intra-and extrahepatic biliary tract and a dilated Wirsung duct (Figure 1).

Upper endoscopy revealed a tumor protrusion in the descending duodenum segment with normal mucous coating. Laboratory examinations showed a total bilirubin of $5.58 \mathrm{mg} / \mathrm{dl}$, AST $110 \mathrm{U} / \mathrm{L}$, ALT $251 \mathrm{U} / \mathrm{L}$, amylasemia

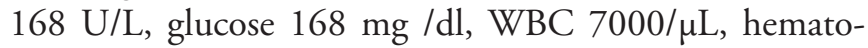
crit $38.9 \%$. After appropriate preoperative preparation, surgery was preformed under general anesthesia with oro- 


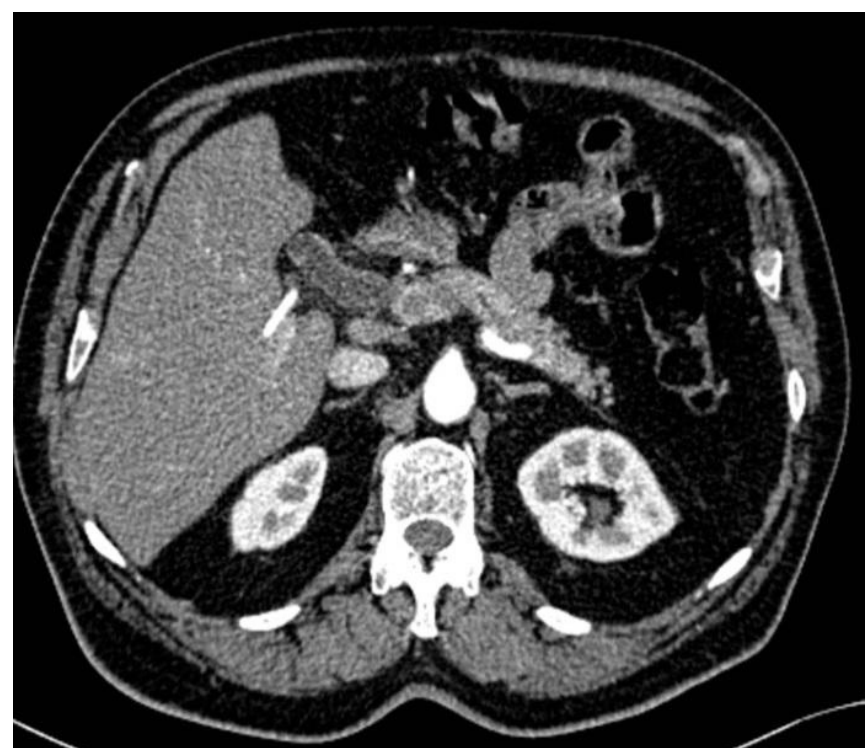

Fig. 1. Computed tomography appearance

tracheal intubation. Intraoperative a tumoral process was revealed, having about 3-4 centimeters, belonging to the ampulla of Vater and common bile duct stones. After declaring operability, cephalic duodeno-pancreatectomy and coledocolitotomy (for stone about 5 milimetres) were performed. For the pancreatico-digestive anastomosis we prefered a pancreatico-gastric procedure. For this purpose the remnant pancreatic stump was mobilized about 3 centimeters. We applied two threads on the pancreatic stump and the Wirsung duct was cannulated with a perforated plastic tube and fixed with resorbable thread (Figure 2).

The tube was inserted about 5 centimeters, leaving about 2 centimeters outside the duct. At the level of the posterior gastric wall corresponding to the pancreatic stump a transverse incision was made, about 2 centimeters, to ensure

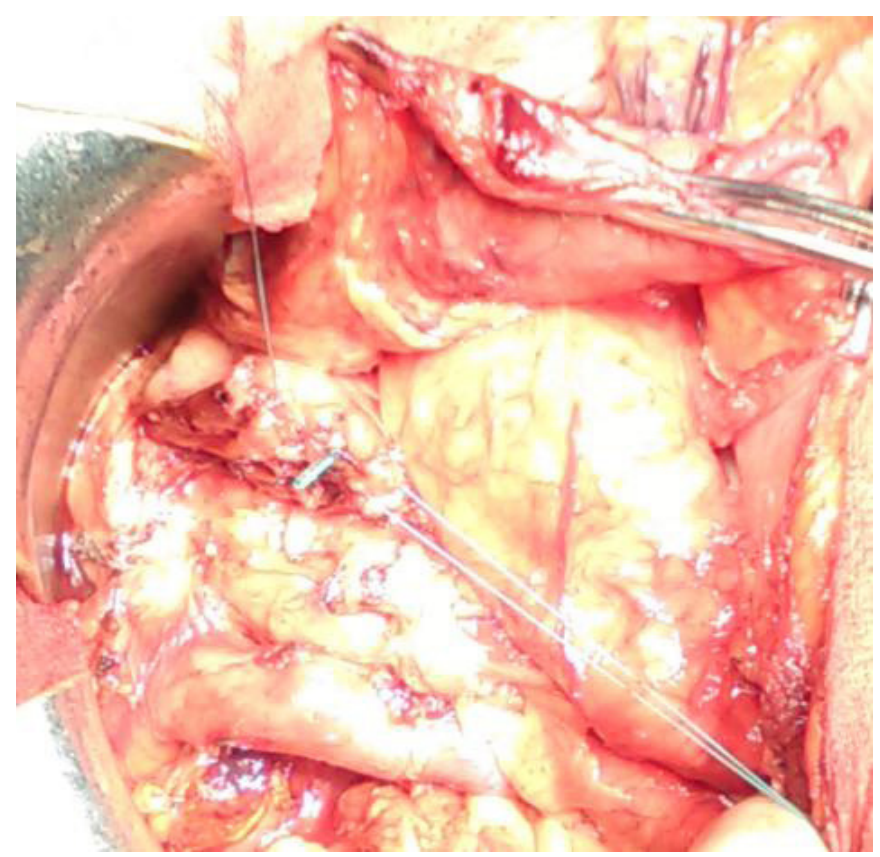

Fig. 2. The appearance of pancreatic stump prepared for anastomosis

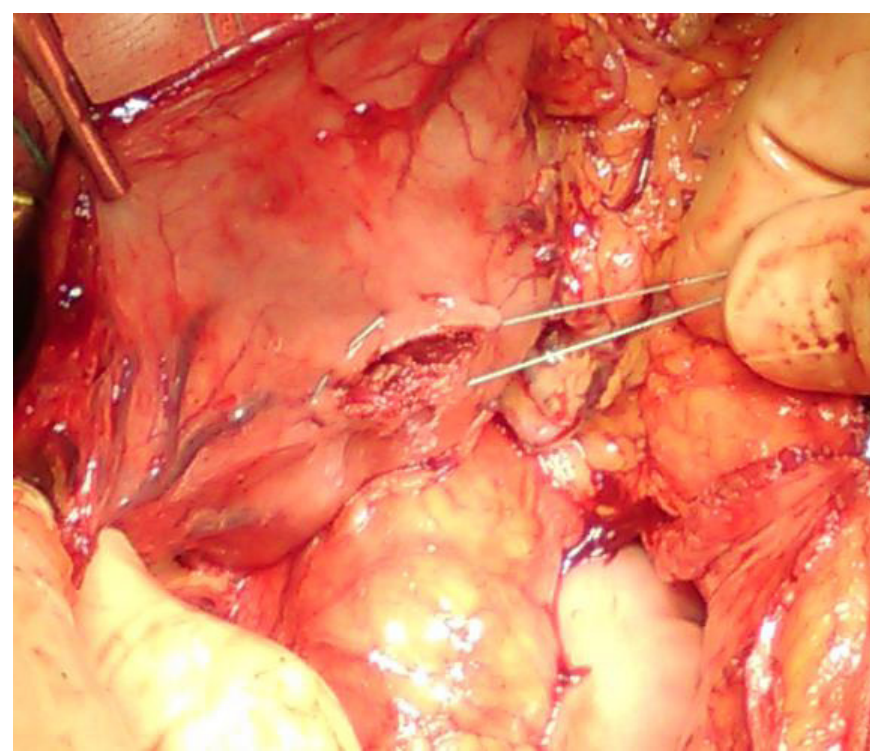

Fig. 3. Posterior gastric wall (seromuscular) purse string.

a close adherence of pancreatic stump to the gastric wall. Around the incision we placed a purse string thread involving one centimeter of the sero-muscular layer (Figure 3).

Through the gastric section we performed another purse string thread, involving one centimeter of the corresponding mucosa incision on the posterior gastric wall (Figure 4).

The pancreatic stump was gently pulled in the stomach, so that it protuded about 2 centimeters above the posterior gastric wall. The external purse string was tighten first, followed by internal one (Figure 5).

Other anastomoses performed were termino-lateral hepatico-jejunostomy and latero-lateral gastro-enteroanastomosys. The intervention ended with the placement of double subhepatic drainage. The postoperative evolution

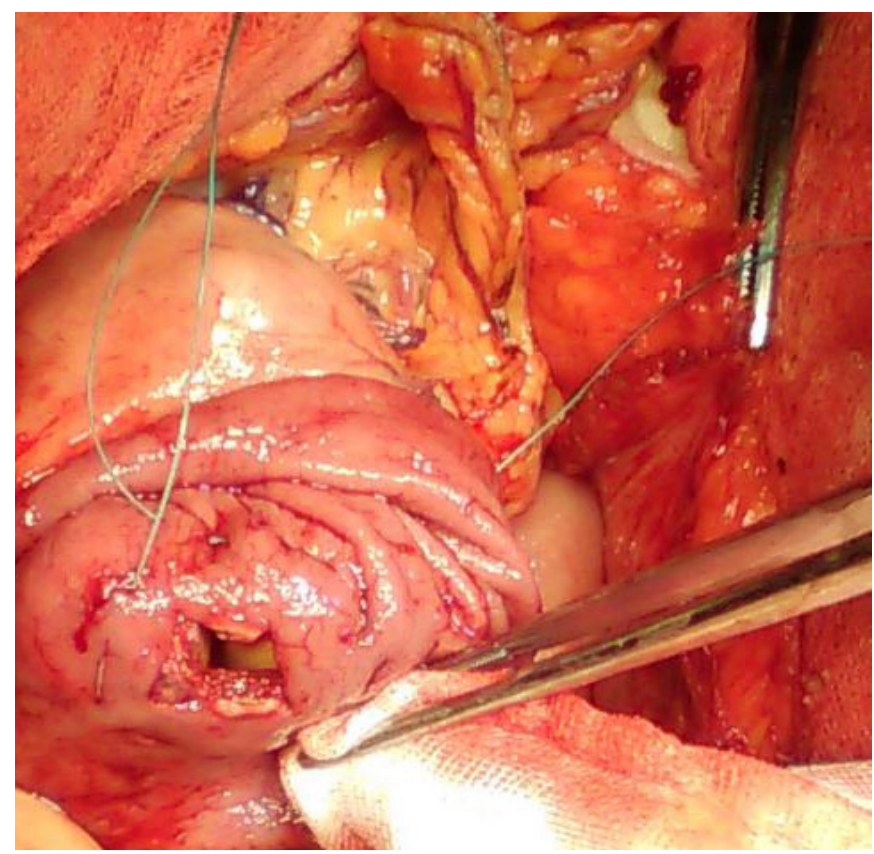

Fig. 4. Posterior gastric wall (mucosa) purse string. 


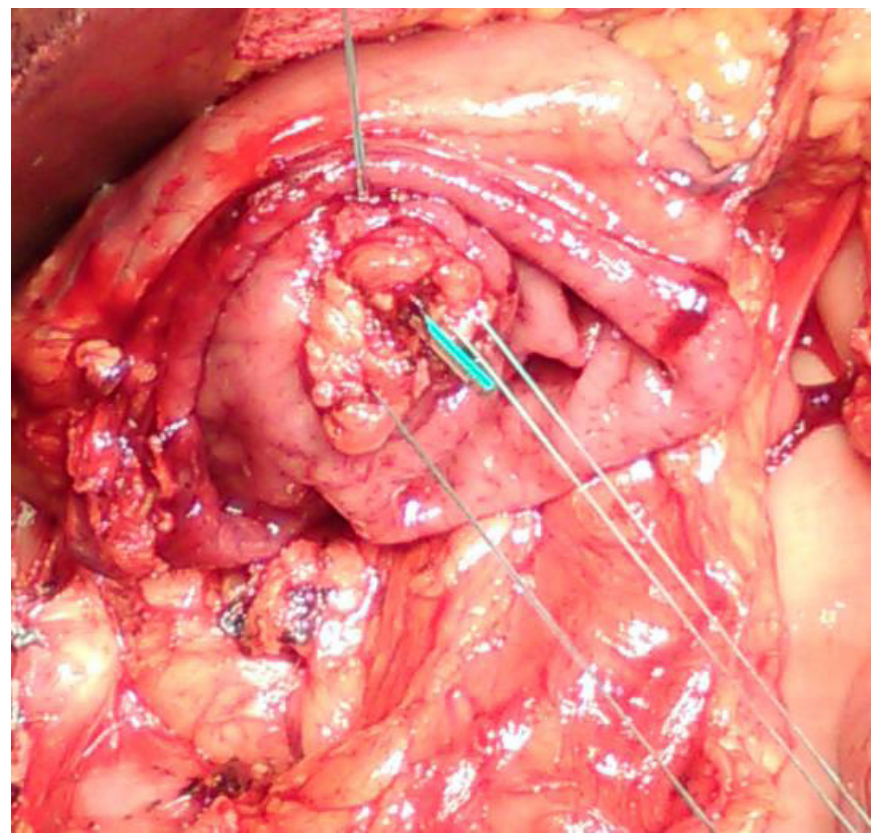

Fig. 5. The pancreatic stump pulled in the stomach.

was favorable, the patient was discharged on the 12 th postoperative day. Histopathology revealed a poorly differentiated ampullary adenocarcinoma (G3), with invasion of duodenal submucosa and metastases in 5 of 7 peripancreatic lymph nodes and 4 of 4 periduodenal lymph nodes; pT2 N1 M0.

\section{Discussion}

The method of pancreatico-digestive anastomosis after cephalic duodeno-pancreatectomy continues to provoke controversy, some surgeons supporting the pancreatico-jejunal anastomosis, while others preferring pancreatico-gastric reconstruction $[10,11,12,13]$. The main cause of morbidity and mortality after cephalic duodeno-pancreatectomy remains pancreatic fistula, especially in the so-called "soft pancreas." Although there were no differences in the rate of pancreatic fistula in four randomized prospective studies $[5,6,7]$, pancreatico-gastric anastomosis is preferred by many surgeons because of several advantages. Primarily, stomach pancreas anastomosis is facilitated by the proximity of the two organs. Moreover, the thickness of the gastric wall and its good vascularization makes it a good suture material. In addition, the large stomach cavity provides good adaptation of pancreatic stump, compared to the smaller diameter of the proximal jejunum. In addition gastric acidity inhibits the activation of pancreatic enzymes, making it a protective factor against the occurrence of pancreatic fistula. Another advantage of pancreatico-gastric anastomosis is the possibility to resolve by endoscopy an eventual anastomosis dehiscence.

Originally described by Waughn and Clagett in 1946 [14] pancreatico-gastric anastomosis has seen many technical variants in recent years. All the variants so far, including duct-to-mucosa anastomosis, the use of trans-pancreatic "U" threads [15], using 2 purse strings [16] or the com- bined use of one purse string and 2 " $U$ " threads [17], to name just a part, aimed at reducing the pancreatic fistula after cephalic duodeno-pancreatectomy. Until now we can say that there is no "gold standard" technique for this.

In the case presented, after cephalic duodeno-pancreatectomy we performed a pancreatic-gastric reconstruction. The remnant pancreatic stump was prepared approximately 3 centimeters, separating it from the splenic vessels and a polyethylene tube was inserted about 5 centimeters in the Wirsung duct. We used two purse string threads, at the level of posterior gastric seromuscular wall and one at the corresponding mucosa layer. After pulling the pancreatic stump into the stomach through the posterior gastric wall incision, the external purse string thread was tightened followed by the internal one. Peng et al published in 2009 an article describing a similar technique but performed a posterior gastric wall seromuscular excision corresponding to the pancreatic stump, making a mucosal tube that coats the pancreatic stump in the stomach [16]. Ohigashi et al in 2008 described a pancreatico-gastric anastomosis using 4-6 " $U$ " threads which pass through the pancreatic stump and posterior gastric wall [15]. Combining the two techniques, Bartsch described in 2012 a variant in applying two transpancreatic " $U$ " threads through the pancreatic stump and a purse string thread posterior to the gastric wall [17]. A procedure similar to that described by Peng was published in 2013 by Addeo et al. In this alternative the technique used was the placement of 2 purse string threads to the gastric posterior seromuscular layer and one purse string to the gastric posterior mucosal layer. [18]. Zhu et al describe a technique similar to that published by Peng, in which the two threads in purse string from the seromuscular and gastric mucosa and are applied through the posterior gastric approach [19].

All these techniques that avoid or minimize the use of trans-pancreatic suture threads, avoiding pancreatic tissue trauma, are particularly useful especially in the case of socalled "soft pancreas." In the present case we used 2 purse string threads, one internal to the mucosa and one external to the seromuscular layer, avoiding crossing " $U$ " threads through the pancreatic parenchyma as in the procedure described by Bartsch or seromusculara excision of the stomach, like in procedures described by Zhu and Peng.

The rate of pancreatic fistulas in procedures that use purse string for pancreatic-gastric anastomosis is reduced. Thus, Addeo reports a rate of $8 \%$ of pancreatic fistulas [18], Bartsch 8.6\% [17] 2.2\% Zhu [19] and Peng 0\% [17].

Given its simplicity, feasibility and low rate of pancreatic fistulas in techniques using purse string for pancreaticgastric anastomosis, we believe that these procedures must be placed in the therapeutic arsenal of surgeons, being especially useful for so-called "soft pancreas ".

\section{Conclusions}

The technique described is a safe, fast and feasible procedure. Pancreatic fistula rate is reduced using this technique 
due to the avoidance of crossing threads through the pancreatic tissue.

\section{Acknowledgement}

This paper is supported by the Sectoral Operational Programme Human Resources Development (SOP HRD), financed from the European Social Fund and by the Romanian Government under the contract number POSDRU/159/1.5/S/133377/

\section{References}

1. Reid-Lombardo KM, et al. Pancreatic anastomotic leakage after pancreaticoduodenectomy in 1,507 patients: a report from the Pancreatic Anastomotic Leak Study Group. J Gastrointest Surg 2007;11:1451-1458.

2. Pratt WB, et al. Clinical and economic validation of the International Study Group of Pancreatic Fistula (ISGPF) classification scheme. Ann Surg 2007;245:443-451.

3. Butturini $\mathrm{G}$, et al. Complications after pancreaticoduodenectomy: the problem of current definitions. J Hepatobiliary Pancreat Surg 2006;13:207211.

4. Deoliveira ML, et al. Assessment of complications after pancreatic surgery: a novel grading system applied to 633 patients undergoing pancreaticoduodenectomy. Ann Surg 2006;244: 931

5. Yeo CJ, et al. A prospective randomized trial of pancreaticogastrostomy versus pancreaticojejunostomy after pancreaticoduodenectomy. Ann Surg 1995;222:580-592

6. Duffas JP, et al. A controlled randomized multicenter trial of pancreatogastrostomy or pancreatojejunostomy after pancreatoduodenectomy. Am J Surg 2005;189:720-729.

7. Bassi C, et al. Reconstruction by pancreaticojejunostomy versus pancreaticogastrostomy following pancreatectomy: results of a comparative study. Ann Surg 2005; 242:767.

8. Fernandez-Cruz, et al. Pancreatogastrostomy with gastric partition after pylorus-preserving pancreatoduodenectomy versus conventional pancreatojejunostomy: a prospective randomized study. Ann Surg 2008;248:930-938

9. Aranha GV, et al. A comparison of pancreaticogastrostomy and pancreaticojejunostomy following pancreaticoduodenectomy. J Gastrointest Surg 2003;7:672-682.

10. Adams, DB. The pancreatic anastomosis: the danger of a leak, which anastomotic technique is better? J Gastrointest Surg 2009;13:11821183.

11. Bassi C, et al. Duct-to-mucosa versus end-to-side pancreaticojejunostomy reconstruction after pancreaticoduodenectomy: results of a prospective randomized trial. Surgery 2003; 134:766-771.

12. Berger AC., et al. Does type of pancreaticojejunostomy after pancreaticoduodenectomy decrease rate of pancreatic fistula? A randomized, prospective, dual-institution trial. J Amm Coll Surg 2009;208:738-747

13. Cameron JL, et al. One thousand consecutive pancreaticoduodenectomies. Ann Surg 2006; 244:10.

14. Waugh JM, Clagett OT. Resection of the duodenum and head of the pancreas for carcinoma; an analysis of thirty cases. Surgery 1946;20:224.

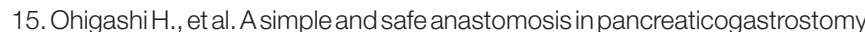
using mattress sutures. Am J Surg 2008;196:130-134.

16. Peng SY, et al. A pancreas suture-less type \| binding pancreaticogastrostomy. Zhonghua wai ke za zhi [Chinese journal of surgery] 2009;47:1764-1766.

17. Bartsch DK, et al. A simple and safe anastomosis for pancreatogastrostomy using one binding purse-string and two transfixing mattress sutures. Int $J$ Surg Oncol 2012;2012:718637

18. Addea P, et al. Double purse-string telescoped pancreaticogastrostomy: an expedient, safe, and easy technique. J Am Coll Surg 2013;216:e27.

19. Zhu F, et al. Modified technique of pancreaticogastrostomy for soft pancreas with two continuous hemstitch sutures: a single-center prospective study. J Gastrointest Surg 2013; 17:1306-1311. 